\title{
Microtubule reorganization during mitosis and cytokinesis: lessons learned from developing microgametophytes in Arabidopsis thaliana
}

\author{
Bo Liu*, Chin-Min Kimmy Ho and Yuh-Ru Julie Lee
}

Department of Plant Biology, University of California, Davis, CA, USA

Edited by:

Marisa Otegui, University of Wisconsin at Madison, USA

\section{Reviewed by:}

Yoshinobu Mineyuki, University of

Hyogo, Japan

David Twell, University of Leicester, UK

\section{*Correspondence:}

Bo Liu, Department of Plant Biology University of California, 1 Shields

Avenue, Davis, CA 95616, USA.

e-mail: bliu@ucdavis.edu
In angiosperms, mitosis and cytokinesis take place in the absence of structurally defined microtubule-organizing centers and the underlying mechanisms are largely unknown. In the spindle and phragmoplast, microtubule reorganization depends on microtubule-interacting factors like the $\gamma$-tubulin complex. Because of their critical functions in cell division, loss-of-function mutations in the corresponding genes are often homozygous or sporophytic lethal. However, a number of mutations like gem1, gcp2, and nedd1 can be maintained in heterozygous mutants in Arabidopsis thaliana. When mutant microspores produced by a heterozygous parent undergo pollen mitosis I, they are amenable for phenotypic characterization by fluorescence microscopy. The results would allow us to pinpoint at specific functions of particular proteins in microtubule reorganization that are characteristic to specific stages of mitosis and cytokinesis. Conclusions made in the developing microgametophytes can be extrapolated to somatic cells regarding mechanisms that regulate nuclear migration, spindle pole formation, phragmoplast assembly, and cell division plane determination.

Keywords: microgametophyte, microtubules, phragmoplast, spindle, Arabidopsis thaliana
Higher plant cells lack structurally defined microtubule-organizing centers (MTOCs) like the centrosome present in cells of animals, most algae, and basal land plants. Consequently, mitosis is executed by acentrosomal spindles. In fact, the absence of the centrosome is accompanied by great plasticity of the spindle morphology observed in various somatic and reproductive cells in different plant species (Palevitz, 1993). Following mitosis, cytokinesis is brought about by the phragmoplast, an evolutionary landmark first appeared in advanced green algae (Pickett-Heaps, 1975; Graham et al., 2000), which gives rise to the cell plate resulted from the fusion of Golgi-derived vesicles. Plant cytokinesis is primarily dependent on microtubules (MTs), unlike that in animal and fungal cells which employ the actomyosin contractile ring (Guertin et al., 2002). Studies of mitosis and cytokinesis in animals have been motivated by their direct implications in understanding causes of diseases like cancers and developing therapeutic strategies. While some aspects of mitosis and cytokinesis are expected to be conserved in plant and animal cells (Lloyd and Chan, 2006), one should not anticipate that results of animal cell-based work would allow us to elucidate all molecular mechanisms that regulate plant cell division.

Successful cell division is the fundamental basis of growth and reproduction among all organisms. Thus, serious disturbance of cell division would result in lethality. Critical functions of MT-associated factors often can be revealed by phenotypic analysis of loss-of-function mutations that may provide clues about their specific roles in mitosis and cytokinesis. Perplexingly, sporophytic lethal mutations could not be brought to the homozygous status in somatic cells so that it becomes challenging to perform cytological experiments in order to elucidate how MTs may be disorganized in mutant cells. Earlier dissection of specific roles of proteins of interest in mitosis was often aided by microinjections of antibodies, which was aimed for blocking the functions of target proteins (Scholey, 1998). Alternatively, gene expression could be transiently brought down by approaches like RNA interference in cultured cells or early developing embryos of animals (Srayko et al., 2005; Goshima et al., 2007). In addition, spindles can be assembled in vitro using the oocyte/egg extract of the frog Xenopus (Gard and Kirschner, 1987; Heald et al., 1996). In this in vitro system, depletion of individual factors by antibodies would allow one to investigate their functions in mitosis (Wang and Zheng, 2011).

In flowering plants, it is technically challenging to establish a system like the Xenopus egg extract due to difficulties in protein preparation. Microinjection also relies on precise instrumentation and sophisticated skills. Nevertheless, microinjection of fluorescently labeled proteins and antibodies has been achieved in the stamen hair cells of the spiderwort Tradescantia virginiana (Zhang et al., 1990; Vos et al., 2000). Unfortunately, not much if any is known about the genetic makeup of spiderwort. Tobacco BY-2 cells also have been an extremely valuable system for studies of MTs and cell division in plants (Nagata and Kumagai, 1999). However, the complex tobacco genome has not been fully sequenced and annotated so that biochemical results often are not met with genetic dissections. In contrast, the completed Arabidopsis thaliana and rice genomes have allowed us to closely examine genes encoding tubulins and proteins that interact with MTs (Guo et al., 2009). However, to date we only know functions of a handful of proteins that regulate MT organization in plant cells partly due to limited availability of homozygous mutants for corresponding genes. 
When a loss-of-function mutation is created for an essential gene, it is ideally kept in the heterozygote state and hoped to be transmitted through sexual reproduction. One would expect that fundamental architecture of the mitotic and meiotic spindles is at least similar if not identical. When a heterozygous plant carry out meiosis, a lethal mutation may be lost after the wild type and mutant alleles are separated into two different nuclei if they are separated in two cells. The model plant $A$. thaliana allows us to overcome this potential obstacle. In many plants like lily, when the microsporocyte/microspore mother cell undergoes meiosis, successive cytokinesis takes place after each round of nuclear division. In A. thaliana, however, the nuclear division of meiosis I is not followed by cytokinesis (McCormick, 1993). Instead, simultaneous cytokinesis takes place at the end of meiosis II to produce four microspores (Yang et al., 2003). When a heterozygous plant produces microspores, the mutant allele (a) would be included in two of the four microspores, while the other two contain the wild type allele (A) (Figure 1A). Under such a circumstance, viable microspores carrying the mutations would be produced by the heterozygous parent. Thus, meiosis of the heterozygous diploid microsporocyte would result in two wild type haploid microspores and two mutant ones, and all microspores inherit the cytoplasm of the heterozygous parent.

In a life cycle exhibiting alternation of generations, the haploid microspore undergoes mitosis to produce the microgametophyte. In angiosperms, the microspore undergoes an asymmetrical cell division (pollen mitosis I) to produce two cells of different fates, concomitantly the pollen grain/young microgametophyte is being

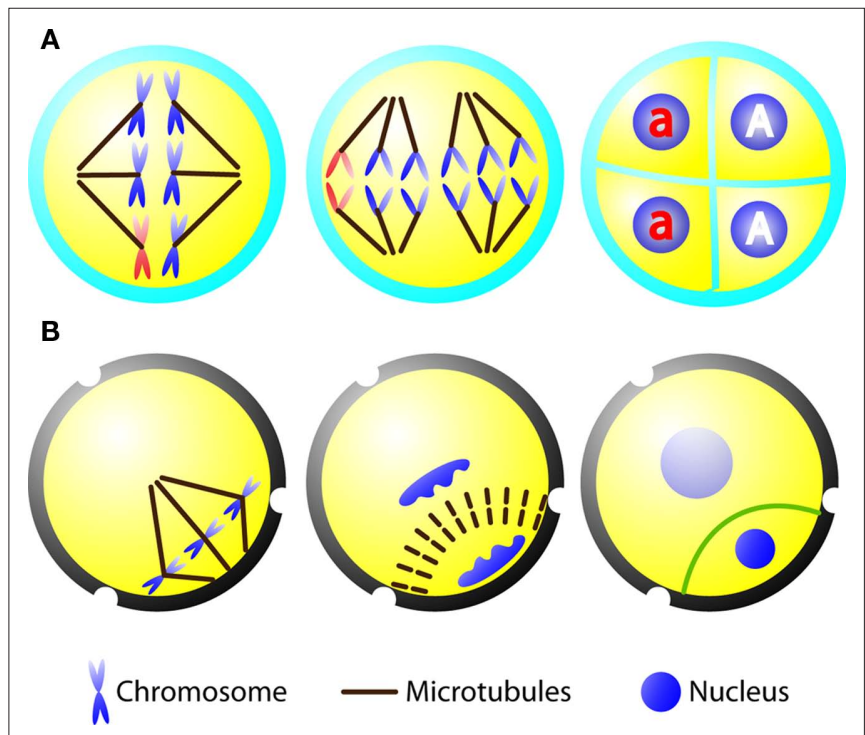

FIGURE 1 | Diagrams depicting microsporocyte meiosis and pollen mitosis I in A. thaliana. (A) Sequential diagrams showing the spindle of meiosis I (left), two spindles of meiosis II (middle), and tetrad (right) containing two wild type microspores (highlighted as " $A$ ") and two mutant ones (highlighted as " $a$ "). The chromosomes carrying the mutation are shown in red. (B) Diagrams demonstrating pollen mitosis I with asymmetrically positioned spindle (left) and phragmoplast (middle). A curved cell plate (green line) is formed to result in a lens-shaped generative cell with densely packed chromatin and a larger vegetative cell with loosely packed chromatin (right). developed (Abrash and Bergmann, 2009; Borg and Twell, 2010). A smaller lens-shaped cell is the generative cell which divides to form two sperm cells via one round of mitosis (pollen mitosis II), and the larger cell is the vegetative cell which will produce the pollen tube upon pollen germination. The asymmetrical pollen mitosis I is preceded by migration of the microspore nucleus toward the cell cortex (McCormick, 1993). This mitotic event, as in other reproductive cells, does not present a preprophase band (PPB) MT array. Upon the breakdown of the nuclear envelope, chromosomes are attached to the mitotic spindle which is placed asymmetrically in the cytoplasm (Figure 1B). In addition, the spindle itself is asymmetrical as shown by two unequal half spindles (Terasaka and Niitsu, 1990; Zonia et al., 1999; Oh et al., 2010c). The peripheral spindle pole is typically wide and nearly in contact with the plasma membrane. In dividing microspores of orchids, an MT array named generative pole MT system (GPMS) was observed between the plasma membrane and the prophase microspore nucleus prior to the assembly of the spindle (Brown and Lemmon, 1991). However, this MT array was not observed in dividing microspores expressing a green fluorescent protein (GFP)-tubulin marker in A. thaliana and tobacco (Oh et al., 2010a,c). Instead a perinuclear MT network extends to the cell cortex (Oh et al., 2010c). This MT network and the GPMS may contribute to establishing the peripheral half spindle. Conversely, the interior pole of the spindle is more or less focused, pointing at the center of the cytoplasm (Figure 1B). In somatic cells, the formation of the mitotic spindle is often linked to or influenced by the PPB (Ambrose and Cyr, 2008). It is known that plant and animal cells employ more than one pathway to establish the bipolar spindle MT array (Lloyd and Chan, 2006). Hence, pollen mitosis I could serve as an excellent model for investigating PPBindependent mechanisms underlying spindle formation. The fact that the spindle of pollen mitosis I is relative short $(\sim 5 \mu \mathrm{m})$ and asymmetrically positioned is also intriguing in regarding to how the length of the mitotic spindle is regulated, a question often asked by animal cell biologists (Goshima and Scholey, 2010).

At late anaphase and telophase during pollen mitosis I, MTs are reorganized into the bipolar phragmoplast array between two reforming nuclei. Remarkably, the phragmoplast expands centrifugally in a curved fashion as if some guidance is provided (Figure 1B). Consequently, a curved cell plate is formed, separating the generative and vegetative cells. In the absence of the PPB, how does the phragmoplast know where to fuse at the parental plasma membrane? In other words, what is the positional signal to be recognized by the expanding phragmoplast? It was proposed that the cell is a domain of cytoplasm formed around the nucleus, and the cytoplasmic domain is established by nuclear position and the interaction between MTs radiating from the neighboring nuclear envelopes (Brown and Lemmon, 1992; Pickett-Heaps et al., 1999). Radial MTs were suggested to measure the cytoplasmic domain and trigger the centrifugal expansion of the phragmoplast (Brown and Lemmon, 1992). It has been known for decades that cell division events like pollen mitosis I do not involve the PPB. Hence, $\mathrm{PPB}$-independent mechanisms must regulate cell division plane determination in reproductive cells like the microspores.

Mutations in genes encoding proteins regulating MT organization often are homozygous lethal as reported (Park et al., 1998; Nakamura and Hashimoto, 2009; Zeng et al., 2009). Developing 
microgametophytes serve as excellent models for investigating defects in MT organization brought about by sporophytic lethal mutations. Among four products of meiosis by a heterozygous parent, two wild type ones would serve as the positive control while the mutant spindle and phragmoplast are examined. If a gene is essential for mitosis or cytokinesis, one would expect that gametes could not be produced by developing gametophytes. Intriguingly, for reasons unknown such mutations have been found to be transmitted through either male or female gametes or both with compromised transmission efficiency as reported (Zeng et al., 2009). This is somewhat surprising because proteins essential for cell division would be equally important for gametophyte and sporophyte development. The imperfect transmission efficiency is caused by partial failures in pollen mitosis I. If the mutant microspores completely failed to divide, the corresponding mutation could not have been inherited. The inheritance of the mutation through sexual reproduction suggests that the mitotic phenotype exhibits incomplete penetrance. Such a phenomenon may be due to the presence of the cytoplasm derived from the heterozygous microsporocyte and megasporocyte. Because developing microgametophytes/pollen grains can be isolated from immature anthers, they are amenable for examinations of defects in chromosome segregation, spindle formation, cell plate formation caused by sporophytic lethal mutations. In A. thaliana, immunolocalization of MTs in intact pollen grains was recently made possible (Lee et al., 2007). Moreover, a microgametophyte specific GFP-tubulin marker was introduced in tobacco and $A$. thaliana, so that transgenic microgametophytes are ready for the observation of MT arrays in live cells (Oh et al., 2010a,c).

In the past few years, the microgametophyte has been gradually employed for mutant-based analysis aimed at deciphering mechanisms that regulate the organization of spindle and phragmoplast MT arrays. Earlier genetic studies isolated mutants such as scp and gem 1 that exhibit aberrant microspore divisions (Chen and McCormick, 1996; Park et al., 1998). The gem1 mutant was found to be homozygous lethal because it caused failures in cytokinesis (Twell et al., 2002). Further phenotypic analysis employing the microspore/microgametophyte targeted GFP-tubulin reporter and RNA interference has greatly advanced our understanding the essential functions of the XMAP215/DIS1 ortholog MOR1/ GEM1 in organizing the spindle and phragmoplast MT arrays (Oh et al., 2010c). This study in tobacco revealed that the MOR1/GEM1 ortholog TMBP200 was essential for pre-mitotic nuclear migration by regulating the stability and dynamicity of MTs. It further pinpointed its critical role of in spindle and phragmoplast orientation. In addition, multipolar spindles and branched phragmoplasts were induced upon down regulation of TMBP200 expression (Oh et al., 2010c). Consequently, pollen mitosis I was jeopardized with altered division planes and cell plates, resembling the phenotype exhibited by the gem1 mutant in A. thaliana (Park et al., 1998; Oh et al., 2010c). These phenotypes observed during pollen mitosis I could be extrapolated to mitosis in somatic cells.

For years, plant cell biologists have been attacking the question about how MTs are nucleated to give rise to the spindle and phragmoplast arrays (Murata and Hasebe, 2011). The $\gamma$-tubulin complex is known to be essential for MT nucleation among all eukaryotes (Job et al., 2003). In developing microgametophyte lacking $\gamma$-tubulin or the $\gamma$-tubulin complex protein 2 (GCP2), the spindle MT array collapses and cytokinesis fails to take place (Pastuglia et al., 2006; Nakamura and Hashimoto, 2009). Earlier studies showed that $\gamma$-tubulin is broadly associated with spindle and phragmoplast MT arrays with biases toward MT minus ends (Liu et al., 1993, 1995). How is $\gamma$-tubulin targeted to non-centrosomal sites for MT nucleation? In animal cells, the WD40 protein NEDD1/GCP-WD interacts with the $\gamma$-tubulin complex and directs it to MT nucleation sites (Lüders et al., 2006). A plant NEDD1 relative, albeit sharing low sequence homology to its animal counterparts, definitely functions in MT organization during mitosis (Zeng et al., 2009). When a heterozygous nedd 1 mutant was examined in A. thaliana, defective bipolar spindles were observed in mutant microspores during pollen mitosis I (Figure 2). Unlike short spindles placed near the cortex in the wild type cells, however, the mutant spindle typically lost length control and expanded across the cytoplasm in the dividing cell (Figure 2). The nedd 1 mutation also caused collapsed phragmoplasts with random MT masses between two reforming nuclei that do not exhibit obvious bipolarity (Zeng et al., 2009). Although the nedd1 mutation can be transmitted through sexual reproduction, homozygous mutant seeds were never recovered. Thus, developing microgametophyte became an invaluable material for investigating defects in MT organization upon the loss of proteins like NEDD1. Furthermore, different phenotypes in MT organization exhibited by the nedd1 and gem 1 mutants allow us to distinguish the specific functions of these essential proteins. The work on NEDD1 also has instructed us that it is possible to isolate additional loss-of-function mutations that may affect fundamental MT nucleation activity in the spindle and phragmoplast. Recently, the eight-subunit augmin complex has been demonstrated to mediate centrosome-independent but MT-dependent MT nucleation in animal cells (Goshima and Kimura, 2010). Augmin is believed to bridge the interaction between the $\gamma$-tubulin complex and MTs via the NEDD1 protein (Zhu et al., 2008). Plants produce a few proteins remotely related to augmin subunits. If they were essential for organizing the spindle and phragmoplast MT arrays, it would be difficult to isolate homozygous mutants for the corresponding genes. Investigation of the functions of these putative augmin homologs would be greatly facilitated by examining mutant microgametophytes produced by heterozygous parents once corresponding mutant alleles are isolated.

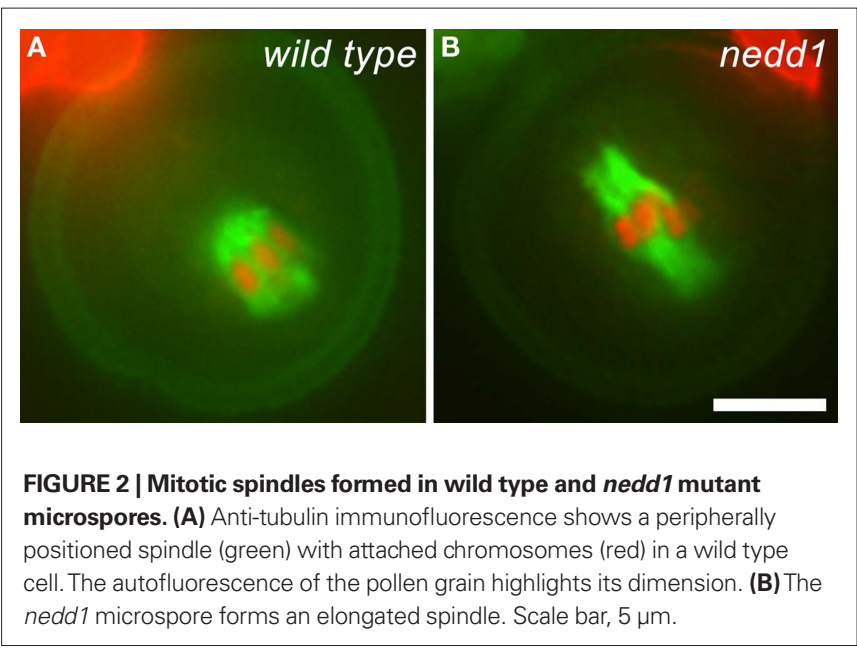


Genetic dissections of microgametophyte development also recovered novel components of the cytokinesis machinery such as the Fused kinase TIO which localizes to the phragmoplast midline (Oh et al., 2005). Again, TIO's role in cytokinesis is not limited to the microgametophyte. The kinase exerts a specific role in cell plate expansion among all examined cytokinesis (Oh et al., 2005). Another work resulted in isolating microgametophyte specific SCP protein which may act as a nuclear factor specifically regulating the division plane position and orientation in pollen mitosis I (Oh et al., 2010b).

The elegant pollen mitosis I has long been a model for the study of asymmetrical cell division (Otegui and Staehelin, 2000). The rich genetic resources gathered by the A. thaliana community have offered us an unprecedented opportunity to elucidating functions of proteins that regulate mitosis and cytokinesis in angiosperms. Earlier microscopic observations of splendid mitotic events in models like lily endosperm cells and onion guard mother cells will gradually be embraced by the discoveries of specific roles of novel MT-interacting proteins. There are many outstanding questions regarding dynamic reorganization of spindle and phragmoplast MTs (Liu et al., 2011; Malcos and Cyr, 2011). For example, it is unknown how the perinuclear MT array becomes reorganized into the bipolar spindle in the absence

\section{REFERENCES}

Abrash, E. B., and Bergmann, D. C. (2009). Asymmetric cell divisions: a view from plant development. Dev. Cell 16, 783-796.

Ambrose, J. C., and Cyr, R. (2008). Mitotic spindle organization by the preprophase band. Mol. Plant 1, 950-960.

Borg, M., and Twell, D. (2010). Life after meiosis: patterning the angiosperm male gametophyte. Biochem. Soc. Trans. 38, 577-582.

Brown, R. C., and Lemmon, B. E. (1991). Pollen development in orchids 3. A novel generative pole microtubular system predicts unequal pollen mitosis. J. Cell Sci. 99, 273-281.

Brown, R. C., and Lemmon, B. E. (1992). Cytoplasmic domain: a model for spatial control of cytokinesis in reproductive cells of plants. EMSA Bull.22, 48-53.

Chen, Y. C., and McCormick, S. (1996). Sidecar pollen, an Arabidopsis thaliana male gametophytic mutant with aberrant cell divisions during pollen development. Development 122,3243-3253.

Gard, D. L., and Kirschner, M. W. (1987). Microtubule assembly in cytoplasmic extracts of Xenopus oocytes and eggs. J. Cell Biol. 105, 2191-2201.

Goshima, G., and Kimura,A. (2010). New look inside the spindle: microtubuledependent microtubule generation within the spindle. Curr. Opin. Cell Biol. 22, 44-49.

Goshima, G., and Scholey, J. M. (2010). Control of mitotic spindle length. Annu. Rev. Cell Dev. Biol. 26, 21-57.
Goshima, G., Wollman, R., Goodwin, S. S., Zhang, N., Scholey, J. M., Vale, R. D., and Stuurman, N. (2007). Genes required for mitotic spindle assembly in Drosophila S2 cells. Science 316, 417-421.

Graham, L.E., Cook, M.E., and Busse, J.S. (2000). The origin of plants: body plan changes contributing to a major evolutionary radiation. Proc. Natl. Acad. Sci. U.S.A. 97, 4535-4540.

Guertin, D. A., Trautmann, S., and eukaryotes. Microbiol. Mol. Biol. Rev. 66, 155-178.

Guo, L., Ho, C.-M. K., Kong, Z., Lee, Y.-R. J., Qian, Q., and Liu, B. (2009). Evaluating the microtubule cytoskeleton and its interacting proteins in monocots by mining the rice genome. Ann. Bot. 103, 387-402.

Heald, R., Tournebize, R., Blank, T., Sandaltzopoulos, R., Becker, P., Hyman, A., and Karsenti, E. (1996). Self-organization of microtubules into bipolar spindles around artificial chromosomes in Xenopus egg extracts. Nature 382, 420-425.

Job, D., Valiron, O., and Oakley, B. (2003). Microtubule nucleation. Curr. Opin. Cell Biol. 15, 111-117.

Lee, Y. R. J., Li, Y., and Liu, B. (2007). Two Arabidopsis phragmoplast-associated kinesins play a critical role in cytokinesis during male gametogenesis. Plant Cell 19, 2595-2605.

Liu, B., Hotta, T., Ho, C.-M. K., and Lee, Y. R. J. (2011). "Microtubule organization in the phragmoplast," in The McCollum, D. (2002). Cytokinesis in

of the centrosome. Neither do we know how the phragmoplast MT array expands centrifugally during cytokinesis. Although mutations in genes encoding proteins fundamentally important for MT organization are likely homozygous lethal in sporophytes, they can be inherited in and transmitted through the microgametophyte. Mutant microgametophytes become gateways to elucidating the functions of such proteins because of the accessibility of the mutant cells. Once functions of more proteins are characterized, we expect that pieces of information would be integrated and a network connecting these proteins would be revealed. Ultimately, we would learn from the microgametophyte regarding mechanisms that regulate nuclear migration, chromatin-dependent spindle formation, spindle pole formation, asymmetrical cell division, and cell division plane determination in angiosperms.

\section{ACKNOWLEDGMENTS}

Our work was supported by the National Science Foundation (NSF) under the grant MCB-0920454 and by the U.S. Department of Energy (DOE) under the contract DE-FG02-04ER15554. Any opinions, findings, and conclusions or recommendations expressed in this report are those of the authors and do not necessarily reflect the views of NSF or DOE.

Plant Cytoskeleton, ed. B. Liu (New York: Springer), 207-225.

Liu, B., Joshi, H. C., and Palevitz, B. A. (1995). Experimental manipulation of $\gamma$-tubulin distribution in Arabidopsis using anti-microtubule drugs. Cell Motil. Cytoskeleton 31, 113-129.

Liu, B., Marc, J., Joshi, H. C., and Palevitz, B. A. (1993). A $\gamma$-tubulin-related protein associated with the microtubule arrays of higher plants in a cell cycledependent manner. J. Cell Sci. 104, 1217-1228.

Lloyd, C., and Chan, J. (2006). Not so divided: the common basis of plant and animal cell division. Nat. Rev. Mol. Cell Biol. 7, 147-152.

Lüders, J., Patel, U. K., and Stearns, T. (2006). GCP-WD is a $\gamma$-tubulin targeting factor required for centrosomal and chromatin-mediated microtubule nucleation. Nat. Cell Biol. 8 , 137-147.

Malcos, J. L., and Cyr, R. (2011) "Acentrosomal spindle formation through the heroic age of microscopy: past techniques, present thoughts, and future directions," in The Plant Cytoskeleton, ed. B. Liu (New York: Springer), 187-205.

McCormick, S. (1993). Male gametophyte development. Plant Cell 5, 1265-1275.

Murata, T., and Hasebe, M. (2011). "Microtubule nucleation and organization in plant cells," in The Plant Cytoskeleton, ed. B. Liu (New York: Springer), 81-94.

Nagata, T., and Kumagai, F. (1999). Plant cell biology through the window of the highly synchronized tobacco BY-2 cell line. Methods Cell Sci. 21, 123-127.

Nakamura, M., and Hashimoto, T. (2009). A mutation in the Arabidopsis $\gamma$-tubulin-containing complex causes helical growth and abnormal microtubule branching. J. Cell Sci. 122, 2208-2217.

Oh, S. A., Allen, T., and Twell, D. (2010a). A ticket for the live show: microtubules in male gametophyte development. Plant Signal. Behav. 5, 614-617.

Oh, S. A., Park, K., Twell, D., and Park, S. K. (2010b). The SIDECAR POLLEN gene encodes a microspore-specific LOB/AS2 domain protein required for the correct timing and orientation of asymmetric cell division. Plant J. 64, 839-850.

Oh, S. A., Pal, M. D., Park, S. K., Johnson, J. A., and Twell, D. (2010c). The tobacco MAP215/Dis1-family protein TMBP200 is required for the functional organization of microtubule arrays during male germline establishment. J. Exp. Bot. 61, 969-981.

Oh, S. A., Johnson, A., Smertenko A Rahman, D., Park, S. K., Hussey, P. J. and Twell, D. (2005). A divergent cellular role for the FUSED kinase family in the plant-specific cytokinetic phragmoplast. Curr. Biol. 15, 2107-2111.

Otegui, M., and Staehelin, L. A. (2000). Cytokinesis in flowering plants: more than one way to divide a cell. Curr. Opin. Plant Biol. 3, 493-502.

Palevitz, B.A. (1993). Morphological plasticity of the mitotic apparatus in plants 
and its developmental consequences. Plant Cell 5, 1001-1009.

Park, S. K., Howden, R., and Twell, D. (1998). The Arabidopsis thaliana gametophytic mutation gemini pollen 1 disrupts microspore polarity, division asymmetry and pollen cell fate. Development 125, 3789-3799.

Pastuglia, M., Azimzadeh, J., Goussot, M., Camilleri, C., Belcram, K., Evrard, J. L., Schmit, A. C., Guerche, P., and Bouchez,D. (2006). $\gamma$-Tubulin is essential for microtubule organization and development in Arabidopsis. Plant Cell $18,1412-1425$.

Pickett-Heaps, J. D. (1975). Aspects of spindle evolution. Ann. N. Y. Acad. Sci. 253, 352-361.

Pickett-Heaps, J.D., Gunning, B.E., Brown, R. C., Lemmon, B. E., and Cleary, A. L. (1999). The cytoplast concept in dividing plant cells: cytoplasmic domains and the evolution of spatially organized cell division. Am. J. Bot. 86, 153-172.

Scholey, J. M. (1998). Functions of motor proteins in echinoderm embryos: an argument in support of antibody inhibition experiments. Cell Motil. Cytoskeleton 39, 257-260.
Srayko, M., Kaya, A., Stamford, J., and Hyman, A. A. (2005). Identification and characterization of factors required for microtubule growth and nucleation in the early C. elegans embryo. Dev. Cell 9, 223-236.

Terasaka, O., and Niitsu, T. (1990). Unequal cell division and chromatin differentiation in pollen grain cells. II: microtubule dynamics associated with the unequal cell division. J. Plant Res. 103, 133-142.

Twell, D., Park, S. K., Hawkins, T. J., Schubert, D., Schmidt, R., Smertenko, A., and Hussey, P. J. (2002). MOR1/ GEM1 has an essential role in the plant-specific cytokinetic phragmoplast. Nat. Cell Biol. 4, 711-714.

Vos, J. W., Safadi, F., Reddy, A. S. N., and Hepler, P. K. (2000). The kinesin-like calmodulin binding protein is differentially involved in cell division. Plant Cell 12, 979-990.

Wang, S., and Zheng, Y. (2011). Identification of a novel dynein binding domain in nudel essential for spindle pole organization in Xenopus egg extract. J. Biol. Chem. 286, 587-593.
Yang, C., Spielman, M., Coles, J., Li, Y., Ghelani, S., Bourdon, V., Brown, R., Lemmon, B., Scott, R., and Dickinson, H. (2003). TETRASPORE encodes a kinesin required for male meiotic cytokinesis in Arabidopsis. Plant J. 34, 229-240.

Zeng, C. J., Lee, Y. R., and Liu, B. (2009). The WD40 repeat protein NEDD1 functions in microtubule organization during cell division in Arabidopsis thaliana. Plant Cell 21, 1129-1140.

Zhang, D. H., Wadsworth, P., and Hepler, P. K. (1990). Microtubule dynamics in living dividing plant cells - confocal imaging of microinjected fluorescent brain tubulin. Proc. Nat. Acad. Sci. U.S.A. 87, 8820-8824.

Zhu, H., Coppinger, J. A., Jang, C. Y., Yates, J. R. III, and Fang, G. (2008). FAM29A promotes microtubule amplification via recruitment of the NEDD1-gamma-tubulin complex to the mitotic spindle. J. Cell Biol. 183, 835-848.

Zonia, L., Tupy, J., and Staiger, C. J. (1999). Unique actin and microtubule arrays co-ordinate the differentiation of microspores to mature pollen in
Nicotiana tabacum. J. Exp. Bot. 50, 581-594.

Conflict of Interest Statement: The authors declare that the research was conducted in the absence of any commercial or financial relationships that could be construed as a potential conflict of interest.

Received:30 May 2011;paperpending published: 15 June 2011; accepted: 21 June 2011; published online: 07 July 2011.

Citation: Liu B, Ho C-MK and Lee Y-RJ (2011) Microtubule reorganization during mitosis and cytokinesis: lessons learned from developing microgametophytes in Arabidopsis thaliana. Front. Plant Sci. 2:27. doi: 10.3389/fpls.2011.00027

This article was submitted to Frontiers in Plant Cell Biology, a specialty of Frontiers in Plant Science.

Copyright (๑) $2011 \mathrm{Liu}$, Ho and Lee. This is an open-access article subject to a nonexclusive license between the authors and Frontiers Media SA, which permits use, distribution and reproduction in other forums, provided the original authors and source are credited and other Frontiers conditions are complied with. 